\title{
A Lower Bound for Controlled Lagrangian Particle Tracking Error
}

\author{
Klementyna Szwaykowska and Fumin Zhang
}

\begin{abstract}
Autonomous underwater vehicles are flexible mobile platforms for ocean sampling and surveillance missions. However, navigation of these vehicles in unstructured, highly variable ocean environments poses a significant challenge. Model-based prediction of vehicle position may be used to improve navigation capability, but prediction error exists due to limited resolution and accuracy of flow values obtained from ocean models that calculate flow velocity at discrete grid points. We present a theoretical lower bound on the steadystate error in position prediction for underwater vehicles using ocean model flow data and show that it is determined by the gridsize used by the ocean models. Our conclusions are justified by simulation and data collected during an ocean experiment.
\end{abstract}

\section{INTRODUCTION}

Autonomous Underwater Vehicles (AUVs) are mobile, flexible sensor platforms which have been used in ocean studies. Thanks to their mobility and capability to operate without constant human supervision, they are able to sample ocean conditions with greater spatial and temporal resolution than is generally possible with moored or ship-based sensor platforms [1]. Multiple AUVs may be deployed in an area of interest simultaneously to form a network for more effective detection and measurement of ocean features [4], [5]. However, localization and navigation for autonomous agents in highly unstructured, stochastic ocean flow fields remains a significant challenge in underwater vehicle control [3].

In this paper, we introduce a novel concept of controlled Lagrange particle tracking (CLPT) that compares the motion of real vehicles in the ocean and that of simulated vehicles with dynamics based on input from ocean models. The CLPT error describes discrepancies between simulated vehicle positions and the true positions. We theoretically justify the existence of a lower bound for the steady-state CLPT error, and compute this bound for the simplified case of onedimensional ocean flows. Our theoretical results are shown to agree with experimental data collected during the Adaptive Sampling and Prediction (ASAP) experiment conducted in Monterey Bay in 2006 [11]. In this experiment, a fleet of 10 underwater gliders were deployed to take ocean samples in a $40 \times 20 \mathrm{~km}$ coastal region off Monterey Bay, CA. The gliders followed predetermined trajectories. An onshore computer was used to generate waypoints along the desired trajectories; these were passed to the gliders, which then used onboard controllers to reach the waypoints. The algorithms for waypoint computation were implemented in the glider coordinated control system (GCCS) [15]. The experiment was run alongside "virtual experiments", which utilized flow

School of Electrical and Computer Engineering, Georgia Institute of Technology. Email: klimka,fumin@ece.gatech.edu data from ocean models (ROMS, NCOM, or HOPS [12][14]) to simulate glider trajectories. The simulated gliders were initialized at the precise positions of their physical counterparts (obtained from GPS fixes taken when the gliders surfaced). Both sets of gliders (real and simulated) used the same internal control laws. The theoretical results developed in this paper explain the similarity between the behaviors of CLPT errors based on different ocean models.

The rest of the paper is organized as follows: in section II, we introduce CLPT and in section III we define the CLPT error. In section IV, we derive a lower bound for the CLPT error in a one-dimensional flow field. Section V describes simulation results for CLPT error growth. Conclusions and future work are described in section VI.

\section{Controlled Lagrangian Particle Tracking}

The goal of controlled Lagrangian particle tracking (CLPT) is to study and predict the trajectories of particles moving in ocean currents. In the past, Lagrangian particle tracking (LPT) has been used extensively to study the motions of drifting sensor platforms deployed in the ocean [7]-[10]. One commonly used approach in LPT is to simulate the trajectories of these platforms by integrating along the underlying Eulerian flow field. However, flow values from ocean models are available only at points of a discrete grid (with minimum gridsize of approximately $1.5 \mathrm{~km}$ ); the limited spatial and temporal resolution of the flow data results in discrepancies between the predicted and the observed vehicle trajectories.

In CLPT, we are interested in predicting the motion of vehicles with adjustable velocity inputs. Using an approach analogous to LPT, we model underwater vehicles as point particles moving in a flow field. The velocity $\mathbf{u}$ of the particle relative to the water is treated as a control input. It has been shown that submesoscale (length scale $\lesssim 10 \mathrm{~km}$ ) dynamics of such a particle moving in the ocean can be modeled to first order using the Langevin equation [6]

$$
\begin{aligned}
& \mathrm{d} \mathbf{x}=(\mathbf{F}(\mathbf{x}, t)+\nu+\mathbf{u}(\mathbf{x}, t)) \mathrm{d} t \\
& \mathrm{~d} \nu=-A \nu \mathrm{d} t+\Lambda \mathrm{d} \omega
\end{aligned}
$$

where $\mathbf{x}(t)$ is the position of the particle, $\mathbf{F}(\mathbf{x}, t)$ is the underlying deterministic flow, and $\nu$ represents small-scale stochastic flow variations which are not captured in the flow predictions given by the ocean models. The matrices $A$ and $\Lambda$ are given by

$$
A=\left[\begin{array}{cc}
\frac{1}{\tau} & \Omega \\
-\Omega & \frac{1}{\tau}
\end{array}\right] \quad \Lambda=\left[\begin{array}{cc}
\sigma \sqrt{\frac{2}{\tau}} & 0 \\
0 & \sigma \sqrt{\frac{2}{\tau}}
\end{array}\right]
$$


where $\sigma$ is the variance of the stochastic velocity fluctuations, $\tau$ represents Lagrangian correlation time, and $\Omega$ is the spin parameter. The stochastic input $\omega$ is modeled as zero-mean, Gaussian white noise.

In simulation using ocean models, the vehicles are again modeled as particles, with dynamics described by

$$
\mathrm{d} \mathbf{z}=(\hat{\mathbf{F}}(\mathbf{z}, t)+\mathbf{u}(\mathbf{z}, t)) \mathrm{d} t
$$

where $\mathbf{z}(t)$ is the position of the particle in simulation, and $\hat{F}(\mathbf{z}, t)$ represents the flow estimate obtained from ocean models. Flow predictions from ocean models are given at discrete spatial points; flow values at intermediate locations are obtained by bilinear interpolation of the values at the gridpoints.

\section{THE CLPT ERROR}

We examine the discrepancy between the position of a particle in the environmental simulation $\mathbf{z}(t)$, and the true position $\mathbf{x}(t)$, assuming that the simulated and real particles are initialized at the same point $(\mathbf{x}(0)=\mathbf{z}(0))$.

The position error of the particle is defined as $\mathbf{e}(t)=$ $\mathbf{x}(t)-\mathbf{z}(t)$. We will assume that the simulated and real particles are initialized to the same position $(x(0)=z(0))$ so that $e(0)=0$. The equations for the evolution of the error in time are given by:

$$
\begin{aligned}
\mathrm{d} \mathbf{e}(t) & =[\mathbf{F}(\mathbf{x}, t)+\mathbf{u}(\mathbf{x}, t)-\hat{\mathbf{F}}(\mathbf{z}, t)-\mathbf{u}(\mathbf{z}, t)+\nu] \mathrm{d} t \\
\mathrm{~d} \nu & =-A \nu \mathrm{d} t+\Lambda \mathrm{d} \omega .
\end{aligned}
$$

Defining $\mathbf{f}(\mathbf{z}, t):=\mathbf{F}(\mathbf{z}, t)-\hat{\mathbf{F}}(\mathbf{z}, t)$, the equation for e may be written as:

$$
\mathrm{d} \mathbf{e}(t)=[\mathbf{F}(\mathbf{x}, t)+\mathbf{u}(\mathbf{x}, t)-\mathbf{F}(\mathbf{z}, t)-\mathbf{u}(\mathbf{z}, t)] \mathrm{d} t+[\mathbf{f}(\mathbf{z}, t)-\nu] \mathrm{d} t .
$$

The first term of this equation reflects error growth caused by the error in the simulated position. In practice, the control velocity $\mathbf{u}$ may be designed to cancel the effects of the deterministic component of the flow. Thus, in the ideal case, the first term in (4) is negligibly small for appropriate $\mathbf{u}$. The error growth may therefore be modeled as:

$$
\begin{aligned}
\mathrm{d} \mathbf{e}(t) & =[\mathbf{f}(\mathbf{z}, t)-\nu] \mathrm{d} t \\
\mathrm{~d} \nu & =-A \nu \mathrm{d} t+\Lambda \mathrm{d} \omega
\end{aligned}
$$

with $\mathbf{e}(0)=0$. Note that $\mathbf{f}(\mathbf{z}, t)$ and $\nu$ are mutually independent terms. Taking the expected value of the error, using Ito calculus, gives:

$$
\begin{aligned}
\frac{\mathrm{d} E(\mathbf{e})}{\mathrm{d} t} & =E(\mathbf{f}(\mathbf{z}, t))-E(\nu) \\
\frac{\mathrm{d} \nu}{\mathrm{d} t} & =-A E(\nu) .
\end{aligned}
$$

The equation for $\nu$ represents a stable dynamical system; therefore as $t \rightarrow \infty, E(\nu) \rightarrow 0$, and the system will converge to stable equilibrium points of the equation

$$
\frac{\mathrm{d} E(\mathbf{e})}{\mathrm{d} t}=E(\mathbf{f}(\mathbf{z}, t)) \text {. }
$$

In general $\mathbf{f}$ is an unknown function of position and time, whose values depend on the particular realization of the stochastic ocean flow. Given a particular ocean flow, however, $\mathbf{f}$ is completely deterministic, and the conditional expected value $E(\mathbf{f})=\mathbf{f}$.

Given perfect flow cancellation, the expected position of the real glider remains $E(\mathbf{x}(t))=\mathbf{x}(0)$. Therefore, $E(\mathbf{e}(t))=E(\mathbf{z}(t)-\mathbf{x}(0))$. We can assume, without loss of generality, that the glider starts at the origin, $\mathbf{x}(0)=\mathbf{z}(0)=$ 0 . Then $E(\mathbf{e}(t))$ given by (7) will be equal to $\mathbf{z}(t)$ in the following system:

$$
\mathrm{d} \mathbf{z}=\mathbf{f}(\mathbf{z}, t) \mathrm{d} t, \quad \mathbf{z}(0)=0
$$

where $\mathbf{f}(\mathbf{z}, t):=\hat{\mathbf{F}}(\mathbf{z}, t)-\mathbf{F}(\mathbf{z}, t)$. For simplicity, we will ignore the time variation of $\mathbf{f}$ and treat it as a function of $\mathbf{z}$ only. We also assume that $\mathbf{f}$ has equilibria; that is, there exits a non-empty set $\mathbf{Z}$ s.t. $f(\mathbf{z}, t)=0 \forall \mathbf{z} \in \mathbf{Z}$.

If we can show that, for a given realization of $\mathbf{f}, \mathbf{z} \rightarrow \mathbf{z}_{e q}$ as $t \rightarrow \infty$, where $\mathbf{z}_{e q}$ is a stable equilibrium of (8), then $\left(\mathbf{z}_{e q}-\mathbf{z}(0)\right)$ is the steady state position error. The CLPT error is defined as the magnitude of the position error, $\mid \mathbf{z}_{e q}-$ $\mathbf{z}(0) \mid$. The expected CLPT error, $E\left(\left|\mathbf{z}_{e q}-\mathbf{z}(0)\right|\right)$, where $E$ is taken over possible realizations of $\mathbf{f}$, gives an estimate of the theoretical steady state position error in estimated position.

Differences over time between the true and simulated glider positions were calculated to find the CLPT position error for the ASAP experiment. A plot of error growth over time, averaged over all the gliders in the experiment, is given in Fig. 1. Note that the qualitative behavior of the error is the same for experiments run using data from different ocean models; the error increases exponentially until it reaches a value approximately twice the size of the grid used by the model, and almost linearly thereafter.

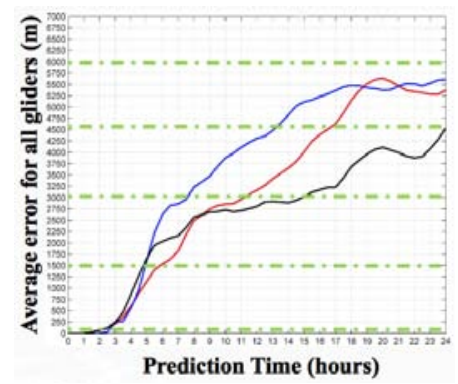

Fig. 1. CLPT error observed in ASAP experiment over a period of 24 hours, averaged over all gliders. The different lines correspond to errors obtained from simulations incorporating flow values from three different ocean models (ROMS, NCOM, and HOPS)

\section{CLPT ERROR FOR ONE-DIMENSIONAL FLOW}

To theoretically compute the CLPT error $E\left(\left|\mathbf{z}_{e q}-\mathbf{z}(0)\right|\right)$ when $\mathbf{z}$ asymptotically converges to $\mathbf{z}_{e q}$, we begin with the case of one-dimensional flow. We use the following setup:

1) The particle is confined to move in one dimension (the domain of $\mathbf{f}$ is $\mathbb{R}$, so $\mathbf{f}$ is now a scalar function, which we denote by $f$; we will denote the scalar $\mathbf{z}$ by $z$ ).

2) We define a grid over $\mathbb{R}$, with uniform gridsize $h$. The ocean model outputs an estimate $\hat{F}$ of the flow $F$ at each gridpoint $z_{k}=k h$. We assume that the values of 
$f(z)=\hat{F}(z)-F(z)$ at the grid points are iid Gaussian white noise variables, which will be denoted $\xi_{k}\left(\xi_{k}=\right.$ $\left.f\left(z_{k}\right)=f(k h)\right)$; and that values of $f$ at intermediate points can be found by taking the linear interpolation of values at grid points. For $z \in\left(z_{k}, z_{k+1}\right)$,

$$
f(z)=\frac{\left(z_{k+1}-z\right) \xi_{k}+\left(z-z_{k}\right) \xi_{k+1}}{h} .
$$

3) The initial conditions are given by $z(0)=x(0)=0$. by

Under these conditions, the equilibria of $f(z)$ are given

$$
z_{e q}=\left(\xi_{k+1} z_{k}-\xi_{k} z_{k+1}\right) /\left(\xi_{k+1}-\xi_{k}\right) .
$$

Lemma 4.1: The position $z_{e q}$ in (10) is a stable equilibrium of (8) under conditions (1)-(3) if and only if $\xi_{k}>0$ and $\xi_{k+1}<0$.

Proof: A sufficient and necessary condition for $z_{e q}$ to be a stable equilibrium is that it satisfies $\partial f / \partial z\left(z_{e q}\right)=\left(\xi_{k+1}-\right.$ $\left.\xi_{k}\right) / h<0$. Thus $z_{e q}$ is a stable equilibrium of (8) if and only if $\xi_{k+1}-\xi_{k}<0$.

Suppose that $\xi_{k}>0$ and $\xi_{k+1}<0$. Clearly, $\xi_{k+1}-\xi_{k}<0$, and we have a stable equilibrium.

Conversely, suppose that $z_{e q}$ is a stable equilibrium, so that $\xi_{k+1}-\xi_{k}<0$ holds. Note that eq. (9) only holds on the interval $\left(z_{k}, z_{k+1}\right)$; that is, $z_{k}<z_{e q}<z_{k+1}$ must be satisfied. Using the equation for $z_{e q}$, we get:

$$
\begin{aligned}
z_{k} & <\frac{\xi_{k+1} z_{k}-\xi_{k} z_{k+1}}{\xi_{k+1}-\xi_{k}}<z_{k+1} \\
z_{k}\left(\xi_{k+1}-\xi_{k}\right) & >\xi_{k+1} z_{k}-\xi_{k} z_{k+1}>z_{k+1}\left(\xi_{k+1}-\xi_{k}\right)
\end{aligned}
$$

where we have used the fact that $\xi_{k+1}-\xi_{k}<0$ to get (12). Taking the inequality on the left-hand side of (12), we find that:

$$
\begin{aligned}
z_{k}\left(\xi_{k+1}-\xi_{k}\right) & >\xi_{k+1} z_{k}-\xi_{k} z_{k+1} \\
z_{k} \xi_{k} & <z_{k+1} \xi_{k} .
\end{aligned}
$$

Using $z_{k}=h k$ and $z_{k+1}=z_{k}+h$ to cancel $z_{k} \xi_{k}$ on both sides of the above inequality yields $0<\xi_{k}$. Similarly, taking the inequality on the right-hand side of (12) yields $\xi_{k+1}>0$. This proves the Lemma.

We now calculate $E\left(\left|z_{e q}\right|\right)$, where $z_{e q}$ is a stable equilibrium of (9) such that $z \rightarrow z_{e q}$ as $t \rightarrow \infty$. We use the definition of expectation to write:

$$
E\left(\left|z_{e q}\right|\right)=\int_{0}^{\infty}|z| \rho_{\left|z_{e q}\right|}(z) \mathrm{d} z
$$

where $\rho_{\left|z_{e q}\right|}(z)$ is the probability density function of $\left|z_{e q}\right|$. It is convenient to introduce $\xi_{0}$ as a conditioning variable in (13), so that we get

$$
\begin{aligned}
E\left(\left|z_{e q}\right|\right)= & \int_{0}^{\infty}|z| \rho_{\left|z_{e q}\right|}\left(z \mid \xi_{0}>0\right) P\left(\xi_{0}>0\right) \mathrm{d} z \\
& +\int_{0}^{\infty}|z| \rho_{\left|z_{e q}\right|}\left(z \mid \xi_{0}<0\right) P\left(\xi_{0}<0\right) \mathrm{d} z
\end{aligned}
$$

Since $\xi_{k}$ are normally-distributed random variables with zero mean, $P\left(\xi_{k}>0\right)=\frac{1}{\sqrt{2 \pi \sigma^{2}}} \int_{0}^{\infty} e^{z^{2} / 2 \sigma^{2}} \mathrm{~d} z=1 / 2$, thus, in the first term, $P\left(\xi_{0}>0\right)=1 / 2$ (similarly, in the second term, $\left.P\left(\xi_{0}<0\right)=1 / 2\right)$, so that

$$
\begin{aligned}
E\left(\left|z_{e q}\right|\right)=\frac{1}{2} \int_{0}^{\infty} & |z| \rho_{\left|z_{e q}\right|}\left(z \mid \xi_{0}>0\right) \mathrm{d} z \\
& +\frac{1}{2} \int_{0}^{\infty}|z| \rho_{\left|z_{e q}\right|}\left(z \mid \xi_{0}<0\right) \mathrm{d} z
\end{aligned}
$$

The calculation of $E\left(\left|z_{e q}\right|\right)$ proceeds through the following three claims:

Claim 4.1: If $\xi_{0}>0$, then $z_{e q}>0$, and moreover, $z_{e q} \in\left(z_{k}, z_{k+1}\right)$ where $z_{k+1}$ is the position corresponding to the first negative value of $\xi$; that is, $\xi_{j}>0$ for all $j \in\{1,2, \ldots, k\}$, and $\xi_{k+1}<0$.

Proof: By the definition in (9), $f$ is a continuous, piecewise affine function.

By continuity, $f(0)=\xi_{0}>0$ implies that $f>0$ in some sufficiently small neighborhood $\epsilon$ of 0 . Thus $z$ cannot become negative, since $d z / d t=f(z)>0$ for all $z \in \epsilon$; so $\lim _{t \rightarrow \infty} z(t)=z_{e q}>0$.

Since $f$ is piecewise affine, $f>0$ on any interval $\left(z_{k}, z_{k+1}\right)$ with $\xi_{k}, \xi_{k+1}>0$. At the same time, $f$ must have a zero-crossing $z_{e q}$ on any interval $\left(z_{k}, z_{k+1}\right)$ with $\xi_{k}>0$ and $\xi_{k+1}<0$. By Lemma 4.1, $z_{e q}$ is a stable equilibrium of (8).

Claim 4.2: If $\xi_{0}<0$, then $z_{e q}<0$, and moreover, $z_{e q} \in\left(z_{-k}, z_{-k+1}\right)$ where $z_{-k}$ is the point corresponding to the first positive value of $\xi$, that is, $\xi_{j}<0$ for all $j \in\{-1,-2, \ldots,-k+1\}$, and $\xi_{-k}>0$. 4.1.

Proof: The proof exactly parallels the proof of Claim

Claim 4.3: Under the assumptions of the one-dimensional problem, we have

$$
\int_{0}^{\infty}|z| \rho_{\left|z_{e q}\right|}\left(z \mid \xi_{0}>0\right) \mathrm{d} z=\int_{-\infty}^{0}|z| \rho_{\left|z_{e q}\right|}\left(z \mid \xi_{0}<0\right) \mathrm{d} z=\frac{3 h}{2} .
$$

Therefore, $E\left(\left|z_{e q}\right|\right)=3 h / 2$.

Proof: The statement $\int_{0}^{\infty}|z| \rho_{\left|z_{e q}\right|}\left(z \mid \xi_{0}>0\right) \mathrm{d} z=$ $\int_{-\infty}^{0}|z| \rho_{\left|z_{e q}\right|}\left(z \mid \xi_{0}<0\right) \mathrm{d} z$ follows from the symmetry of the Gaussian distribution.

We will now show that $E\left(\left|z_{e q}\right|\right)=3 h / 2$. Equation (15) for the expected value of $\left|z_{e q}\right|$ may be written as:

$$
E\left(\left|z_{e q}\right|\right)=\int_{0}^{\infty}|z| \rho_{\left|z_{e q}\right|}\left(z \mid \xi_{0}>0\right) \mathrm{d} z .
$$

This integral in turn can be split up as follows:

$$
\begin{aligned}
\sum_{k=0}^{\infty} \int_{z_{k}}^{z_{k+1}} z \rho_{\left|z_{e q}\right|}\left(z \mid \xi_{0}>0, z_{e q} \in\left(z_{k}, z_{k+1}\right)\right) \mathrm{d} z \\
\times P\left(z_{e q} \in\left(z_{k}, z_{k+1}\right) \mid \xi_{0}>0\right) .
\end{aligned}
$$

By Claim 4.1, $P\left(z_{e q} \in\left(z_{k}, z_{k+1}\right) \mid \xi_{0}>0\right)$ is simply the probability that $\xi_{1}, \ldots, \xi_{k}>0$ and $\xi_{k+1}<0$.

Because the values of $\xi$ are independent, $P\left(\xi_{1}, \ldots, \xi_{k}>\right.$ $\left.0, \xi_{k+1}<0\right)=\left(\prod_{i}^{k} P\left(\xi_{i}>0\right)\right) P\left(\xi_{k+1}<0\right)$. Because $\xi_{i}$ are iid zero-mean Gaussian variables, $P\left(\xi_{i}>0\right)=1 / 2$ for all $i$ (similarly, $\left.P\left(\xi_{k+1}<0\right)=1 / 2\right)$. Using this, and 
applying a change of variables $u=z-z_{k}$, we can write (16) as:

$$
\sum_{k=0}^{\infty}\left[\frac{1}{2}\right]^{k+1} \int_{0}^{h}\left(u+z_{k}\right) \rho_{|u|}\left(u \mid \xi_{0}>0,\left(z_{e q}-z_{k}\right) \in(0, h)\right) \mathrm{d} u .
$$

Suppose we know that $z_{e q}$ lies in the interval $\left(z_{k}, z_{k}+h\right)$. Define $u_{e q}=z_{e q}-z_{k}$ in this interval. Using (10), we can write $u_{e q}$ as:

$$
u_{e q}=\frac{h \xi_{k}}{\xi_{k}-\xi_{k+1}} .
$$

To calculate the integral in (17), we must first find $\rho_{u_{e q}}\left(u \mid u_{e q} \in(0, h)\right)$. To this end, consider the following map:

$$
\phi: \mathbb{R}^{2} \rightarrow \mathbb{R}^{2}, \phi\left(\xi_{k}, \xi_{k+1}\right)=\left(u_{e q}, v\right)
$$

where

$$
\begin{aligned}
u_{e q} & =\frac{h \xi_{k}}{\xi_{k}-\xi_{k+1}} \\
v & =\xi_{k} .
\end{aligned}
$$

The inverse map is well-defined and is given by:

$$
\phi^{-1}(\vec{w})=\left(v, v-h v / u_{e q}\right)=\left(\xi_{k}, \xi_{k+1}\right) .
$$

Let $\vec{\xi}$ denote the doublet $\left(\xi_{k}, \xi_{k+1}\right)$, and $\vec{w}$ denote $\left(u_{e q}, v\right)$. The distribution of $\vec{w}$ is given by:

$$
\tilde{\rho}(\vec{w})=\frac{1}{\left|\operatorname{det} \frac{\partial \phi}{\partial \xi}\right|} \rho\left(\phi^{-1}(\vec{w})\right)
$$

where

$$
\frac{\partial \phi}{\partial \xi}=\left(\begin{array}{cc}
-\frac{h \xi_{k+1}}{\left(\xi_{k}-\xi_{k+1}\right)^{2}} & \frac{h \xi_{k}}{\left(\xi_{k}-\xi_{k+1}\right)^{2}} \\
1 & 0
\end{array}\right)
$$

which gives

$$
\left|\operatorname{det} \frac{\partial \phi}{\partial \xi}\right|=\left|-\frac{h \xi_{k}}{\left(\xi_{k}-\xi_{k+1}\right)^{2}}\right|=\frac{h \xi_{k}}{\left(\xi_{k}-\xi_{k+1}\right)^{2}} .
$$

Substituting (25) into eq. (23) and using (22), we have:

$$
\tilde{\rho}(\vec{w})=\frac{h v}{u_{e q}^{2}} \rho\left(v, v-h v / u_{e q}\right) .
$$

Since $\xi_{k}$ and $\xi_{k+1}$ are Gaussian white noise variables and $\xi_{k}>0$ and $\xi_{k+1}<0$ is given, we have:

$$
\begin{aligned}
\rho\left(\xi_{k}\right) & =\frac{2}{\sqrt{2 \pi \sigma^{2}}} \exp \left\{-\frac{\xi_{k}^{2}}{2 \sigma^{2}}\right\} U\left(\xi_{k}\right) \\
\rho\left(\xi_{k+1}\right) & =\frac{2}{\sqrt{2 \pi \sigma^{2}}} \exp \left\{-\frac{\xi_{k+1}^{2}}{2 \sigma^{2}}\right\} U\left(-\xi_{k+1}\right)
\end{aligned}
$$

where $U(\cdot)$ denotes the unit step function.

Because the values of $\xi$ are iid, the joint probability distribution $\rho(\vec{\xi})=\rho\left(\xi_{k}\right) \rho\left(\xi_{k+1}\right)$. Thus, eq. (26) may be written as:

$$
\tilde{\rho}(\vec{w})=\frac{2 h v}{\pi \sigma^{2} u_{e q}^{2}} e^{-\frac{v^{2}\left(1+\left(1-\frac{h}{u_{e q}}\right)^{2}\right)}{2 \sigma^{2}}} U(v) U\left(v\left(h / u_{e q}-1\right)\right) .
$$

Taking the marginal distribution over $v$ gives:

$$
\begin{aligned}
\rho\left(u_{e q}\right) & =\int_{-\infty}^{\infty} \tilde{\rho}(\vec{w}) \mathrm{d} v \\
& =\frac{2 h}{\pi u_{e q}^{2}} \int_{0}^{\infty} \frac{v}{\sigma^{2}} e^{-\frac{1+\left(1-h / u_{e q}\right)}{2 \sigma^{2}} v^{2}} U\left(h / u_{e q}-1\right) \mathrm{d} v \\
& =\frac{2 h}{\pi\left(u_{e q}^{2}+\left(h-u_{e q}\right)^{2}\right)} U\left(h / u_{e q}-1\right)
\end{aligned}
$$

where we have used a change of variables given by

$$
\tau=\left(1+\left(1-h / u_{e q}\right)^{2}\right) v^{2} / 2 \sigma^{2} .
$$

Eq. (29) is the distribution function in (17). Plugging in the above result for $\rho_{u_{e q}}(u)=\rho_{\left|z_{e q}-z_{k}\right|}\left(u \mid\left(z_{e q}-z_{k}\right) \in(0, h)\right)$ in (17), we get:

$$
\sum_{k=0}^{\infty}\left[\frac{1}{2}\right]^{k+1} \int_{0}^{h}\left(u+z_{k}\right) \frac{2 h}{\pi\left(u^{2}+(h-u)^{2}\right)} \mathrm{d} u .
$$

Using integration tables, we find that

$$
\begin{aligned}
\int_{0}^{h} u \frac{2 h}{\pi\left(u^{2}+(h-u)^{2}\right)} \mathrm{d} u & =\frac{h}{2} \\
\int_{0}^{h} z_{k} \frac{2 h}{\pi\left(u^{2}+(h-u)^{2}\right)} \mathrm{d} u & =z_{k},
\end{aligned}
$$

so that

$$
\begin{aligned}
\sum_{k=0}^{\infty} & \left(\frac{1}{2}\right)^{k+1} \int_{0}^{h}\left(u+z_{k}\right) \frac{2 h}{\pi\left(u_{e q}^{2}+\left(h-u_{e q}\right)^{2}\right)} \mathrm{d} z \\
& =\sum_{k=0}^{\infty}\left(\frac{1}{2}\right)^{k+1} \frac{h}{2}+\sum_{k=0}^{\infty}\left(\frac{1}{2}\right)^{k+1} z_{k} \\
& =\frac{3 h}{2} .
\end{aligned}
$$

This proves Claim 4.3.

We now extend the above result to the case where the particle's initial position does not coincide with a particular gridpoint (that is, we drop condition 3). Suppose that the particle's initial position is $z(0) \in\left(z_{k}, z_{k+1}\right]$. There are then 4 possible cases, depending on the values of $\xi_{k}=f\left(z_{k}\right)$ and $\xi_{k+1}=f\left(z_{k+1}\right)$ :

1) $\xi_{k}>0$ and $\xi_{k+1}>0$,

2) $\xi_{k}<0$ and $\xi_{k+1}>0$,

3) $\xi_{k}>0$ and $\xi_{k+1}<0$,

4) $\xi_{k}<0$ and $\xi_{k+1}<0$.

Let $c$ be a random variable which labels the above cases; $c$ takes values in the set $\{1,2,3,4\}$, with $P(c=i)=\frac{1}{4}$ for all $i$. The expected value of $\left|z_{e q}-z(0)\right|$ in this case may be calculated using conditional expectations:

$$
E\left(\left|z_{e q}-z(0)\right|\right)=\frac{1}{4} \sum_{i=1}^{4} E\left(\left|z_{e q}-z(0)\right| \mid c=i\right) .
$$

Claim 4.4: The expected value of $\left|z_{e q}-z(0)\right|$ is independent of initial conditions, that is,

$$
E\left(\left|z_{e q}-z(0)\right|\right)=3 h / 2
$$


for any $z(0)$.

Proof: We proceed by calculating $E\left(\mid z_{e q}-z(0) \| c=\right.$ i) for each case in turn.

To begin, consider case 1) $\left(\xi_{k}>0\right.$ and $\left.\xi_{k+1}>0\right)$. By monotonicity of $f$ on $\left(z_{k}, z_{k+1}\right), f(z)>0$ for all $z \in\left(z_{k}, z_{k+1}\right]$; it is clear, then, that the particle moves in the positive z-direction until it passes $z_{k+1}$; the expected distance to the nearest equilibrium is then

$$
E\left(z_{e q}-z(0)\right)=E\left(z_{e q}-z_{k+1}\right)+z_{k+1}-z(0)
$$

where $z_{k+1}>0$ is given. Shifting the origin to $z_{k+1}$ and using Claim 4.3 gives $E\left(z_{e q}-z_{k+1}\right)=3 h / 2$. Plugging this back into eq. (35) gives

$$
E\left(\left|z_{e q}-z(0)\right| \mid c=1\right)=3 h / 2+\left(z_{k+1}-z(0)\right) .
$$

Case 4) $\left(\xi_{k}<0\right.$ and $\left.\xi_{k+1}<0\right)$ is analogous to case 1$)$; this time however, $f(z)<0$ for all $z \in\left(z_{k}, z_{k+1}\right]$. The particle moves in the negative z-direction until it passes $z_{k}$, therefore

$$
E\left(\left|z_{e q}-z(0)\right| \mid c=4\right)=\left(z(0)-z_{k}\right)+3 h / 2 .
$$

Next consider case 2) $\left(\xi_{k}<0\right.$ and $\left.\xi_{k+1}>0\right)$. Here we can calculate the expectation as follows:

$$
\begin{aligned}
& E\left(\mid z_{e q}-z(0) \| c=2\right)= \\
& E\left(\mid z_{e q}-z(0) \| c=2, f(z(0))>0\right) P(f(z(0))>0) \\
& +E\left(\left|z_{e q}-z(0)\right| \mid c=2, f(z(0))<0\right) P(f(z(0))<0) .
\end{aligned}
$$

If $f(z(0))>0$, then $f(z)>0$ for all $z \in\left(z(0), z_{k+1}\right]$; so the particle moves in the positive direction until it passes $z_{k+1}$. As in case 1), the expected value of $\left|z_{e q}-z(0)\right|$ is then $3 h / 2+\left(z_{k+1}-z(0)\right)$. If, on the other hand, $f(z(0))<0$, then $f(z)<0$ for all $z \in\left(z_{k}, z(0)\right)$, and the particle moves in the negative direction until it passes $z_{k}$. As in case 4), the expected value of $\left|z_{e q}-z(0)\right|$ is then $\left(z(0)-z_{k}\right)+3 h / 2$. Eq. (38) can therefore be rewritten as:

$$
\begin{aligned}
& E\left(\left|z_{e q}-z(0)\right| \mid c=2\right) \\
& =\left(\frac{3 h}{2}+\left(z_{k+1}-z(0)\right)\right) P(f(z(0))>0) \\
& \quad+\left(\frac{3 h}{2}+\left(z(0)-z_{k}\right)\right)(1-P(f(z(0))>0)) \\
& =\left(\frac{3 h}{2}+z(0)-z_{k}\right)+\left(h-2\left(z(0)-z_{k}\right)\right) P(f(z(0))>0) .
\end{aligned}
$$

It remains only to calculate the probabilities $P(f(z(0))>$ $0)$ and $P(f(z(0))<0)=1-P(f(z(0))>0)$. Let $y_{0}:=$ $z(0)-z_{k}\left(y_{0} \in(0, h]\right)$. Using eq. (9), we can write:

$$
\begin{aligned}
& P\left(f\left(y_{0}\right)>0 \mid \xi_{k}<0, \xi_{k+1}>0\right) \\
& =P\left(\frac{y_{0} \xi_{k+1}+\left(h-y_{0}\right) \xi_{k}}{h}>0 \mid \xi_{k}<0, \xi_{k+1}>0\right) \\
& =P\left(\xi_{k+1}>\frac{y_{0}-h}{y_{0}} \xi_{k} \mid \xi_{k}<0, \xi_{k+1}>0\right) .
\end{aligned}
$$

This can be calculated, using the Gaussian distribution of the $\xi$ and the fact that $\xi_{k}<0$ and $\xi_{k+1}>0$, as:

$$
\begin{aligned}
& P\left(\xi_{k+1}>\frac{y_{0}-h}{y_{0}} \xi_{k} \mid \xi_{k}<0, \xi_{k+1}>0\right) \\
& =\int_{-\infty}^{0} \operatorname{erfc}\left(\frac{y_{0}-h}{y_{0} \sqrt{2 \sigma^{2}}} s\right) \frac{2}{\sqrt{2 \pi \sigma^{2}}} e^{-\frac{s^{2}}{2 \sigma^{2}}} \mathrm{~d} s .
\end{aligned}
$$

Lastly, consider case 3) $\left(\xi_{k}>0\right.$ and $\left.\xi_{k+1}<0\right)$. By Lemma 1, there is a stable equilibrium $z_{e q} \in\left(z_{k}, z_{k+1}\right)$ where $f\left(z_{e q}\right)=0$. By monotonicity of $f$ within one interval, we have that if $f(z(0))<0$, then $z(0)>z_{e q}$; and if $f(z(0))>0$, then $z_{e q}>z(0)$. Therefore,

$$
\begin{aligned}
E & \left(\mid z_{e q}-z(0) \| c=3\right) \\
= & E\left(z_{e q}-z(0)\right) P(f(z(0))>0) \\
& +E\left(z(0)-z_{e q}\right) P(f(z(0))<0) \\
= & \left(z_{k}+h / 2-z(0)\right) P(f(z(0))>0) \\
& +\left(z(0)-\left(z_{k}+h / 2\right)\right)(1-P(f(z(0))>0)) \\
= & 2\left(\left(z_{k}+h / 2\right)-z(0)\right) P(f(z(0))>0) \\
& -\left(\left(z_{k}+h / 2\right)-z(0)\right)
\end{aligned}
$$

where $P(f(z(0))>0)$ denotes

$$
P\left(f(z(0))>0 \mid \xi_{k}>0, \xi_{k+1}<0\right),
$$

which can be calculated analogously to the probability in case 2) (using $y_{0}:=z(0)-z_{k}$ ):

$$
\begin{aligned}
& P\left(f\left(y_{0}\right)>0 \mid \xi_{k}>0, \xi_{k+1}<0\right) \\
& =1-\int_{0}^{\infty} \operatorname{erfc}\left(\frac{h-y_{0}}{y_{0} \sqrt{2 \sigma^{2}}} s\right) \frac{2}{\sqrt{2 \pi \sigma^{2}}} e^{-\frac{s^{2}}{2 \sigma^{2}}} \mathrm{~d} s . \\
& =1-P\left(f\left(y_{0}\right)>0 \mid \xi_{k}<0, \xi_{k+1}>0\right) .
\end{aligned}
$$

Plugging eq. (36),(37),(39), and (42) into (33) gives

$$
E\left(\left|z_{e q}-z(0)\right|\right)=3 h / 2
$$

which is independent of the value of $z(0)$. This proves Claim 4.4 .

\section{Simulation Results}

The results for CLPT error growth can be generalized to two-dimensional flow. It can be shown that an upper bound on minimum CLPT error as $t \rightarrow \infty$ is given by $2.52 h$. The analytical treatment of this case, however, is more involved and will not be handled here. To compare the results of CLPT with observed error growth in the CLPT experiment, CLPT error growth in the two-dimensional case was simulated in Matlab.

The gridsize in the simulation is normalized to unity, and Matlab's "randn" function was used to generate stochastic zero-mean, Gaussian-distributed $z^{1}$ and $z^{2}$-flow components at each gridpoint. A particle was initialized at $(0,0)^{\top}$, and its position over time was computed by integrating the velocity field along its trajectory, using Matlab's "ode23" function. Flow components at each position were calculated using bilinear interpolation of the gridpoint values.

The simulation is run 5000 times. Some sample plots of CLPT error growth for individual runs are shown in Fig. 
??. Fig. ?? shows the CLPT error growth, averaged over all runs. The simulated CLPT error converges to a value of $2.27 \mathrm{~h}$, which agrees with the theoretical value and with observations made during the ASAP experiment.

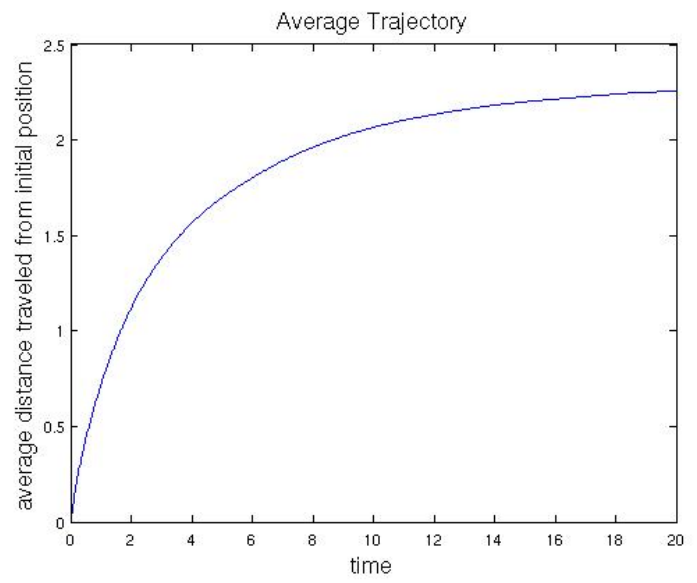

Fig. 2. Simulation of 2-dimensional average CLPT error magnitude growth over time, with gridsize $h$ normalized to 1 .

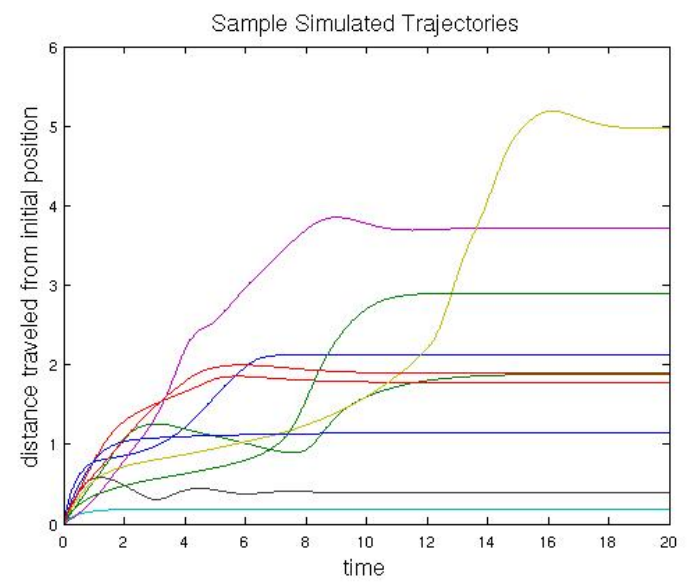

Fig. 3. Sample simulated 2-dimensional CLPT error magnitude growth over time. The grid size is $h=1$.

\section{CONCLUSION AND FUTURE WORK}

The ocean flow in which underwater gliders operate may be decomposed as the sum of a model flow, which can be calculated using ocean models, and an "error flow" which is not captured by the model. This error flow itself has two components: first are small-scale, stochastic components (which we denoted as $\nu$ ) that are not captured by the ocean model due to its limited spatial resolution; and second are larger-scale flows (denoted by $\mathbf{f}$ ) which are caused by missing physics and numerical error within the ocean model. These flows can significantly affect the trajectory-tracking performance of underwater robots. We have shown that using a mathematical model based on Langevin equations for the growth of the CLPT error, it is possible to derive a theoretical lower bound on the magnitude of the robots' model-based position estimation error as $t \rightarrow \infty$.

In our treatment of the CLPT error, we have assumed a particularly simple structure for the error flow in order to make our calculations tractable and develop the idea of CLPT error. Future work will focus on extending these results to more realistic ocean flows, and on applying these results improve the trajectory-tracking performance of underwater gliders.

\section{REFERENCES}

[1] C.C. Eriksen, T.J. Osse, R.D. Light. T. Wen, T. W. Lehman, P.L. Sabin, J.W. Ballard, and A.M. Chidoi. Seaglider: a long-range autonomous underwater vehicle for oceanographic research. Ocean Engineering, IEEE Journal of, 26(4):424-36, 2001.

[2] D.C. Webb, P.J. Simonetti, and C.P. Jones. Slocum: an underwater glider propelled by environmental energy. Oceanic Engineering, IEEE Journal of, 26(4):447-52, 2001.

[3] P. Corke, C. Detweiler, M. Dunbabin, M. Hamilton, D. Rus, and I. Vasilescu. Experiments with Underwater Robot Localization and Tracking. Proc. 2007 IEEE International Conf. on Robotics and Automation, pages 4556-61, 2007.

[4] N.E. Leonard, D. Paley, F. Lekien, R. Sepulchre, D. Fratantoni, and R. Davis. Collective motion, sensor networks, and ocean sampling. Proc. of the IEEE, special issue on the emerging technology of networked control systems, 95:48-74, 2007.

[5] T. Curtin, J. Bellingham, J. Catapovic, and D. Webb. Autonomous oceanographic sampling networks. Oceanography, 6:86-94, 1993.

[6] A.C. Haza, L.I. Piterbarg, P. Martin, T.M. Ozgokmen,A. Griffa. A Lagrangian subgridscale model for particle transport improvement and application in the Adriatic Sea using the Navy Coastal Ocean Model. Ocean Modeling, 17:68-91, 2007.

[7] R.E. Davis, Observing the general-circulation with floats. Deep-Sea Res., 38:531-71, 1991.

[8] W. Owens. A statistical description of the mean circulation and eddy variability in the northwestern Atlantic using SOFAR floats. Progress in Oceanography, 28:257-303, 1991.

[9] P. Richardson. Drifters and floats. Encyclopedia Ocean Studies, 2:76774, 2001.

[10] H. Zhang, M. Prater, and T. Rossby. Isopycnal Lagrangian statistics from the North Atlantic Current RAFOS float observations. J. Geophys. Research, 106:13817-36, 2001.

[11] ASAP: Adaptive Sampling and Prediction. http://www.princeton.edu/ dcsl/asap/.

[12] Y. Chao, Z. Li, J. Farrara, J.C. McWilliams, J. Bellingham, X. Capet, F. Chavez, J.-K. Choi, R. Davis, J. Doyle, D. Frantaoni, P. Li, P. Marchesiello, M.A. Moline, J. Paduan, and S. Ramp. Development, implementation and evaluation of a data-assimilative ocean forecasting system off the central California coast. Deep-Sea Research II, 56:10026, 2007.

[13] I. Shulman, C.R. Wu, J.K. Lewis, J.D. Paduan, L.K. Rosenfeld, J.C. Kindle, S.R. Ramp, and C.A. Collins. High resolution modeling and data assimilation in the Monterey Bay area. Continental Shelf Research, 22(8):1129-51, 2002.

[14] W.G. Leslie, A.R. Robinson, P.J. Haley, O. Logutov, P.A. Moreno, P.F.J. Lermusiaux, and E. Coelho. Verification and training of realtime forecasting of multi-scale ocean dynamics for maritime rapid environmental assessment. Journal of Marine Systems, 69(1-2):3-16, 2008.

[15] D.A. Paley, F. Zhang, D.M. Fratantoni, and N.E. Leonard. Cooperative control systems for ocean sampling: The glider coordinated control system. IEEE Transaction on Control System Technology, 16(4):73544, 2008.

[16] Fumin Zhang, D. M. Fratantoni, D. Paley, J. Lund, N. E. Leonard. Control of Coordinated Patterns for Ocean Sampling. International Journal of Control, 80(7), 1186-99, 2007. 